\title{
Necessary Conditions for Fuzzy Dual Optimal Control Problems
}

\author{
Felix Mora-Camino $^{1 *}$, Fabio Krykhtine ${ }^{1,2}$, Walid El Moudani ${ }^{3}$, Carlos Alberto Nunes Cosenza ${ }^{2}$ \\ ${ }^{1}$ ENAC, Toulouse University, 7 avenue Edouard Belin, 31055 Toulouse, France. \\ 2 Labfuzzy, COPPE, Federal University of Rio de Janeiro, Centro de Tecnologia, Ilha do Fundão, Brazil. \\ 3 Doctorate School of Sciences and Technologies, Libanese University, Tripoli Al-Koubba, Lebanon. \\ * Corresponding author. Tel.: (33)562174000; email:felix.mora@enac.fr \\ Manuscript submitted January 10, 2017; accepted May 28, 2017. \\ doi: 10.17706/ijapm.2017.7.3.182-190
}

\begin{abstract}
This article after introducing fuzzy dual numbers, functions and functionals establishes necessary conditions for the optimization of fuzzy dual functionals before considering fuzzy dual optimal control problems. In a first step an extension of Euler's necessary condition for the extremum of a fuzzy dual functional is established and then necessary conditions for fuzzy dual optimal control are developed.
\end{abstract}

Key words: Euler's condition, fuzzy dual numbers, necessary conditions, optimal control, uncertainty.

\section{Introduction}

Classical optimal control theory based on calculus of variations [1], has played an important role in the design of many modern systems and the understanding of the conditions for their optimal operation. In many situations, real systems are submitted to perturbations which are not completely known, generating uncertainty in their future behavior when controls computed from nominal values are applied. Stochastic optimal control [2] has been developed with acceptable success only in the case of linear systems with a quadratic performance criterion. This approach to be effective needs precise order two statistics for initial state variables and for perturbations along the whole optimization horizon, and it is often not possible to get accurate values for these parameters. Fuzzy optimal control has been also investigated and diverse approaches have been developed mainly based on Takagi-Sugeno (T-S) fuzzy models leading in general to fuzzy control design based on the parallel distributed compensation (PDC) scheme incurring the need for large fuzzy control rule sets [3] . More recently, an attempt to extend the calculus of variations to fuzzy optimal control has been developed in the context of fuzzy mappings parametrized by the left and right functions of their $\alpha$-level sets [4] . In that case a straightforward extension of calculus of variations leads to the establishment of necessary optimality conditions. However, an important limitation of this approach is that fuzzy optimization is based on a partial ordering of fuzzy numbers.

In this paper another approach is developed to cope with fuzzy optimal control. Here a connection is established between dual numbers encountered in the design and analysis of kinematics for mechanical systems [5] and triangular fuzzy numbers, i.e, real intervals for which total orders can be adopted. Here the dual part of dual numbers is associated with the basis of a triangular fuzzy number and different total orders can be considered between these fuzzy numbers [6]. Then fuzzy dual functions, fuzzy dual functionals and fuzzy dual differential equations are introduced. This open the way to establish necessary conditions for the extremum of fuzzy dual functionals and through the introduction of a fuzzy dual 
Hamiltonian, for the extremum of fuzzy dual optimal control problems.

\section{Fuzzy Dual Numbers}

Here we introduce the basic definitions concerning dual numbers and fuzzy dual numbers while some elements of dual number calculus are considered. More details about fuzzy dual calculus can be obtained in [7]. Then total, partial, weak and strong orders between fuzzy dual numbers are introduced.

\subsection{Dual Numbers and Fuzzy Dual Numbers}

The set of dual numbers $\Omega$ is the set of $R^{2}$ with specific addition $(+)$ and multiplication $(\cdot)$ laws given by:

$\forall\left(x_{1}, y_{1}\right),\left(x_{2}, y_{2}\right) \in \Omega:\left(x_{1}, y_{1}\right)+\left(x_{2}, y_{2}\right)=\left(x_{1}+x_{2}, y_{1}+y_{2}\right)$ and $\left(x_{1}, y_{1}\right) \cdot\left(x_{2}, y_{2}\right)=\left(x_{1} x_{2}, x_{1} y_{2}+x_{2} y_{1}\right)$

The set $\Omega$ has a structure of an unity commutative ring with respect to these two laws. Its unitary element is $(1,0)$. The dual number $\varepsilon=(0,1)$ is nilpotent of order two with respect to multiplication, then $\Omega$ has divisors of $(0,0)$ and it is not an integral ring. The subset of $\Omega,\{(x, 0) \mid x \in R\}$, is a sub-ring of $\Omega$ and is isomorph to $R$. The adopted notation for a dual number $(x, y)$ of $\Omega$ is in this paper is $x+\varepsilon y$ where $\varepsilon^{2}=0$ and the zero element $(0,0)$ is written $\tilde{0}$.

To each dual number $a+\varepsilon b$ can be associated a triangular fuzzy number whose membership function is given by:

$$
\begin{array}{crr}
\mu(u)=0 \text { if } \quad u \leq a-|b|, \quad & \mu(u)=(u-a+|b|) /|b| \quad \text { if } \quad a-|b| \leq u \leq a \\
\mu(u)=(a+|b|-u) /|b| \quad \text { if } & a \leq u \leq a+|b|, \quad \mu(u)=0 \text { if } \quad u \geq a+|b|
\end{array}
$$

Then the set of fuzzy dual numbers can be seen as the set $\tilde{\Omega}$ of dual numbers of the form $a+\varepsilon b$ such as $a \in R, b \in R^{+}$where $a$ is the primal part and $|b|$ is the dual part of the fuzzy dual number. Here $a$ is its mean value, the most probable of the fuzzy number according to its triangular membership function, while $2|b|$ is the size of its basis or uncertainty interval. A crisp fuzzy dual number is such as $b$ is equal to zero.

\subsection{Fuzzy Dual Orders}

When considering optimization problems we will be naturally led to compare numbers, here fuzzy dual numbers, and the above definition provides different ways to compare them according to what is pursued through the optimization. Different weak total orders can be defined over $\tilde{\Omega}$, one is relative to the mean value of the fuzzy dual number, the mean order is such as:

$$
x_{1}+\varepsilon y_{1} \underset{\text { mean }}{\leq} x_{2}+\varepsilon y_{2} \Leftrightarrow x_{1} \leq x_{2}
$$

others are relative to their extreme values. The minimal order is such as:

$$
x_{1}+\varepsilon y_{1} \underset{\min }{\leq} x_{2}+\varepsilon y_{2} \Leftrightarrow x_{1}-\left|y_{1}\right| \leq x_{2}-\left|y_{2}\right|
$$

while the maximal order is such as:

$$
x_{1}+\varepsilon y_{1} \underset{\max }{\leq} x_{2}+\varepsilon y_{2} \Leftrightarrow x_{1}+\left|y_{1}\right| \leq x_{2}+\left|y_{2}\right|
$$


These total orders are weak in the sense that they do not allow to compare completely two fuzzy dual numbers and another total order may be added with respect to the degree of uncertainty:

$$
x_{1}+\varepsilon y_{1} \prec x_{2}+\varepsilon y_{2} \Leftrightarrow\left|y_{1}\right| \leq\left|y_{2}\right|
$$

A strong partial order can be defined over $\tilde{\Omega}$ for non-overlapping fuzzy dual numbers:

$$
x_{1}+\varepsilon y_{1} \leq x_{2}+\varepsilon y_{2} \Leftrightarrow x_{1}+\left|y_{1}\right| \leq x_{2}-\left|y_{2}\right|
$$

\subsection{Fuzzy Dual Functions}

Here after introducing classical properties for a function $f$ of a dual variable $x+\varepsilon y$, the differentiability issue is considered, leading to the definition of a fuzzy dual function.

A function $f$ of a dual variable $x+\varepsilon y$ is such as:

$$
f(x+\varepsilon y)=\phi(x, y)+\varepsilon \psi(x, y)
$$

where $\phi$ and $\psi$ are two functions of the real variables $x$ and $y$. This function has a limit equal to $F_{1}+\varepsilon F_{2} \in \Omega$ when $x+\varepsilon y$ goes to $x_{1}+\varepsilon y_{1}$ if and only if:

$$
\lim _{x \rightarrow x_{1}, y \rightarrow y_{1}} \phi(x, y)=F_{1} \quad \text { and } \quad \lim _{x \rightarrow y_{1}, y \rightarrow y_{1}} \psi(x, y)=F_{2}
$$

This function will be continuous at $y_{1}+\varepsilon y_{2}$ if and only if:

$$
\lim _{x \rightarrow x_{1}, y \rightarrow y_{1}} f(x+\varepsilon y)=f\left(y_{1}+\varepsilon y_{2}\right)
$$

Such a function will be differentiable at $x_{1}+\varepsilon y_{1}$ if there exists a dual number $p+\varepsilon q$ and a function $\delta$ of a dual variable $h+\varepsilon l$ such as:

$$
f\left(x_{1}+h, y_{1}+l\right)=f\left(x_{1}, y_{1}\right)+((p+\varepsilon q)+\delta(h+\varepsilon l)) \cdot(h+\varepsilon l) \quad \text { with } \quad \lim _{h \rightarrow 0, l \rightarrow 0} \delta(h+\varepsilon l)=\tilde{0}
$$

Then $p+\varepsilon q$ is the value of the derivative of $f$ at $x_{1}+\varepsilon y_{1}$ and the function of the dual variable defined by $f^{\prime}: \Omega \rightarrow p+\varepsilon q$ is the derivative function of $f$ over $\Omega$. We can write also:

$$
f\left(x_{1}+h, y_{1}+l\right)=f\left(x_{1}, y_{1}\right)+\left(\phi_{x} h+\psi_{y} l\right)+\varepsilon \cdot\left(\psi_{x} h+\psi_{y} l\right)+O^{2}(h+\varepsilon l)
$$

and comparing with (12), it appears that:

$$
p=\phi_{x}=\psi_{y}, \quad q=\psi_{x} \text { and } \phi_{y}=0
$$

and $f$ will be differentiable over a subset of $\Omega$ if and only if at any of its points $\phi_{x}=\psi_{y}$ and $\phi_{y}=0$. Then function $f$ should be of the form [8]:

$$
f(x+\varepsilon y)=\phi(x)+\varepsilon\left(\phi_{x}(x) y+\theta(x)\right)
$$


where $\theta(x)$ is a real valued function. When this last function is zero, we will say that $f$ is a fuzzy dual function and we will write:

$$
\tilde{f}(x+\varepsilon y)=f(x)+\varepsilon y f_{x}(x) \quad \text { for } \quad x \in O \subset R, y \in R
$$

whose fuzzy dual derivative is given by:

$$
\tilde{f}(x+\varepsilon y)^{\prime}=f_{x}(x)+\varepsilon y f_{x x}(x)
$$

Relation (16) can be generalized to a fuzzy dual function $f$ of $n$ dual variables $x_{i}+\varepsilon y_{i}, i=1, \cdots, n$ :

$$
\tilde{f}\left(x_{1}+\varepsilon y_{1}, \cdots, x_{n}+\varepsilon y_{n}\right)=f\left(x_{1}, \cdots ; x_{n}\right)+\varepsilon \sum_{i=1}^{n} y_{i} f_{x_{i}}\left(x_{1}, \cdots, x_{n}\right) \text { for } \quad x_{i} \in O, y_{i} \in R, i=1, \cdots, n
$$

\section{Fuzzy Dual Euler's Equations}

In this section we introduce a fuzzy dual version of the Euler's optimization problem considering first the unconstrained case and then the constrained one where necessary optimality conditions, the fuzzy dual Euler equations are established.

\subsection{Fuzzy Dual Euler Equation: The Unconstrained Case}

Consider a fuzzy dual functional given by:

$$
J(x, y)=\int_{t_{0}}^{t_{f}} f(x+\varepsilon y, \dot{x}+\varepsilon \dot{y}, t) d t
$$

where $x$ and $y$ are real vector functions of $R^{n}$ and $\dot{x}$ and $\dot{y}$ are their derivatives and $f$ is a fuzzy dual function. The problem considered here is to find extremums of $J(x, y)$ which can be rewritten as:

$$
J(x, y)=\int_{t_{0}}^{t_{f}} f(x, \dot{x}, t) d t+\varepsilon \int_{t_{0}}^{t_{f}}\left(f_{x}^{\prime} y+f_{\dot{x}}{ }^{\prime} \dot{y}\right) d t
$$

The fuzzy dual variation of $J$ is now given by:

$$
\delta J=\int_{t_{0}}^{t_{f}}\left(f_{x}^{\prime} \Delta x+f_{\dot{x}}{ }^{\prime} \Delta \dot{x}\right) d t+\varepsilon \int_{t_{0}}^{t_{f}}\left(y^{\prime} f_{x x} \Delta x+\dot{y}^{\prime} f_{\dot{x} \dot{x}} \Delta \dot{x}\right) d t
$$

Considering that $\Delta x(t)$ can be written:

$$
\Delta x(t)=\Delta x\left(t_{0}\right)+\int_{t_{0}}^{t} \Delta \dot{x}(\tau) d \tau
$$

the fuzzy dual variation can be rewritten as:

$$
\delta J=\left[f_{\dot{x}}{ }^{\prime} \Delta x(t)\right]_{t_{0}}^{t_{f}}+\int_{t_{0}}^{t_{f}}\left(f_{x}-\frac{d}{d t} f_{\dot{x}}\right)^{\prime} \cdot \Delta x(t) d t+\varepsilon\left(\left[\dot{y}^{\prime} f_{\dot{x} \dot{x}} \Delta x(t)\right]_{t_{0}}^{t_{f}}-\int_{t_{0}}^{t_{f}}\left(\frac{d}{d t}\left(\dot{y} f_{\dot{x} \dot{x}}\right)^{\prime} \Delta x(t) d t\right)\right.
$$


Considering that the deviations at times $t_{0}$ and $t_{f}$ are equal to zero, the non-integral terms vanish from the above expression:

$$
\delta J=\int_{t_{0}}^{t_{f}}\left(f_{x}-\frac{d}{d t} f_{\dot{x}}\right)^{\prime} \cdot \Delta x(t) d t+\varepsilon \int_{t_{0}}^{t_{f}}\left(\left(f_{x}-\frac{d}{d t} f_{\dot{x}}\right)^{\prime} \Delta y(t)-\frac{d}{d t}\left(\dot{y} f_{\dot{x} \dot{x}}\right)^{\prime} \Delta x(t) d t\right.
$$

A necessary condition to have an extremum for a real valued functional is that its variation must be zero [9]. This can be easily transposed to the present case by considering the mean, the minimal and the maximal extremums of a functional.

\subsection{Constrained Optimization Problems}

Going a step further, we consider the case in which the previous optimization problem is subject to $\mathrm{m}$ constraints according to the fuzzy dual expression:

$$
g(x(t)+\varepsilon y(t), t)=o(t)+\varepsilon z(t)
$$

where $o(t), t \in\left[t_{0}, t_{f}\right]$ is the zero real vector function of $R^{m}$ and $z(t), t \in\left[t_{0}, t_{f}\right]$, is a bounded real function of $R^{m}$, eventually the zero real function. Expression (29) can be rewritten as:

$$
g(x(t), t)=0 \quad \text { and } \quad G_{x}(t) y(t)=z(t) \quad \forall t \in\left[t_{0}, t_{f}\right]
$$

where $G_{x}$ is the Jacobian of $g$. Here we introduce a fuzzy dual Lagrange multiplier written $\lambda+\varepsilon \mu$ where $\lambda \in R^{m}, \mu \in R^{m}$, to build the augmented fuzzy dual functional:

$$
J(x, y, \lambda, \mu)=\int_{t_{0}}^{t_{f}}\left(f(x+\varepsilon y, \dot{x}+\varepsilon \dot{y}, t)+(\lambda+\varepsilon \mu)^{\prime} g(x+\varepsilon y, t)\right) d t
$$

or

$$
J(x, y, \lambda, \mu)=\int_{t_{0}}^{t_{f}} r(x, \dot{x}, \lambda, t) d t+\varepsilon \int_{t_{0}}^{t_{f}} d(x, \dot{x}, y, \dot{y}, \lambda, \mu) d t
$$

with:

$$
r(x, \dot{x}, \lambda, t)=f(x, \dot{x}, t)+\lambda^{\prime} g(x, t)
$$

and

$$
d(x, \dot{x}, y, \dot{y}, \lambda, \mu, t)=\left(f_{x}{ }^{\prime}+\lambda^{\prime} G_{x}\right) y+f_{\dot{x}}^{\prime} \dot{y}+\mu^{\prime} g(x, t)
$$

The fuzzy dual variation of the augmented functional which is associated to deviations $\Delta x, \Delta \dot{x}, \Delta y, \Delta \dot{y}, \Delta \lambda$ and $\Delta \mu$ is now given by:

$$
\delta J=\int_{t_{0}}^{t_{f}}\left(r_{x}{ }^{\prime} \Delta x+r_{\dot{x}}{ }^{\prime} \Delta \dot{x}+g^{\prime} \Delta \lambda\right) d t+\varepsilon \int_{t_{0}}^{t_{f}}\left(d_{x}{ }^{\prime} \Delta x+d_{\dot{x}}{ }^{\prime} \Delta \dot{x}+y^{\prime} G_{x}{ }^{\prime} \Delta \lambda+g^{\prime} \Delta \mu\right) d t
$$

or

$$
\begin{gathered}
\left.\delta J=+\int_{t_{0}}^{t_{f}}\left(r_{x}-\frac{d}{d t}\left(r_{\dot{x}}\right)\right)^{\prime} \Delta x+g^{\prime} \Delta \lambda\right) d t+\varepsilon \int_{t_{0}}^{t_{f}}\left(\left(d_{x}-\frac{d}{d t}\left(d_{\dot{x}}\right)\right)^{\prime} \Delta x+y^{\prime} G_{x}{ }^{\prime} \Delta \lambda+g^{\prime} \Delta \mu\right) d t \\
+\left[r_{\dot{x}}{ }^{\prime} \Delta x(t)\right]_{t_{0}}^{t_{f}}+\varepsilon\left[d_{\dot{x}}{ }^{\prime} \Delta x(t)\right]_{t_{0}}^{t_{f}}
\end{gathered}
$$


Considering again that the deviations at times $t_{0}$ and $t_{f}$ are taken equal to zero and that the considered solutions are feasible, the variation of the augmented functional can be written:

$$
\delta J=\int_{t_{0}}^{t_{f}}\left(r_{x}-\frac{d}{d t}\left(r_{\dot{x}}\right)\right)^{\prime} \Delta x(t) d t+\varepsilon \int_{t_{0}}^{t_{f}}\left(\left(d_{x}-\frac{d}{d t}\left(d_{\dot{x}}\right)\right)^{\prime} \Delta x(t)\right) d t
$$

The necessary condition to have a mean extremum for $J$ is given by the classical Euler equation applied to function $r$ :

$$
r_{x}-\frac{d}{d t}\left(r_{\dot{x}}\right)=0
$$

The necessary conditions to have a minimal extremum for $J$ are given by the augmented Euler equations:

$$
\left(r_{x}-d_{x}\right)-\frac{d}{d t}\left(r_{\dot{x}}-d_{\dot{x}}\right)=0
$$

The necessary conditions to have a maximal extremum for $J$ are given by the augmented Euler equations:

$$
\left(r_{x}+d_{x}\right)-\frac{d}{d t}\left(r_{\dot{x}}+d_{\dot{x}}\right)=0
$$

while conditions (26) must be satisfied.

\section{Fuzzy Dual Optimal Control Problems}

\subsection{Problem Formulation}

In this section we consider a class of optimal control problems where the system to be controlled is subject to perturbations whose uncertainty is imbedded in a fuzzy dual function representing the dynamics of the considered process to be controlled. Let the formulation be given by:

$$
\min _{u \in R^{m}} J(u) \quad \text { with } \quad J(u)=\int_{t_{0}}^{t_{f}} f(z, u, t) d t \quad \text { with } f \in C^{2}
$$

where the state dynamics of the process are such as:

$$
\dot{z}=a(z, u, v+\varepsilon w, t)
$$

where $a \in C^{2}$ with $z=x+\varepsilon y \in \Delta^{n}, u \in R^{m}, v \in R^{p}$ and $w \in R^{p}$ is a fuzzy dual function.

It is supposed here that times $t_{0}$ and $t_{f}$ are given and the initial and final conditions are such as: $x\left(t_{0}\right)=x_{0}, x\left(t_{f}\right)=x_{f}, y\left(t_{0}\right)=y_{0}, y\left(t_{f}\right)$ free. It is supposed that $\underline{v}$ and $\underline{w}$ are given over the interval $\left[t_{0}, t_{f}\right]$. Introducing the Jacobians $A_{x}=\left[\alpha_{i j}\right]=\left[a_{i x_{j}}\right]$ and $A_{v}=\left[\beta_{i k}\right]=\left[a_{i v_{k}}\right]$, the state equation can be rewritten as:

$$
\dot{x}=a(x, u, v, t) \quad \text { and } \quad \dot{y}=A_{x}(x, u, v, t) y+A_{v}(x, u, v, t) w
$$

while the optimization criterion is such as: 


$$
J(u)=\int_{t_{0}}^{t_{f}} f(x, u, t) d t+\varepsilon \int_{t_{0}}^{t_{f}} f_{x}^{\prime} \underline{y} y d t
$$

\subsection{Mean Optimal Control Problem}

mber In that case, the optimal control problem reduces to a classical optimization problem:

$$
\min _{u} \int_{t_{0}}^{t_{f}} f(x, u, t) d t \text { with } \quad \dot{x}=a(x, u, v), \quad x\left(t_{0}\right)=x_{0} \text { and } \quad x\left(t_{f}\right)=x_{f}
$$

Then introducing the classical Hamiltonian function [10], given by:

$$
H=f(x, u, t)+\lambda^{\prime} a(x, u, v, t)
$$

we get the necessary conditions for a mean optimal control solution:

$$
\dot{x}=H_{\lambda}(x, u, \lambda, t) \quad \dot{\lambda}=-H_{x}(x, u, \lambda, t) \quad \text { and } \quad H_{u}(x, u, \lambda, t)=\tilde{0}
$$

with no transversality condition. Let $\left(x^{\text {mean }}, u^{\text {mean }}\right)$ be the solution of the above optimal control problem to which is attached a performance written $J^{\text {mean }}$.

\subsection{Extremal Optimal Control Problems}

sep Here for sake of brevity we treat simultaneously the minimal and maximal extremum problems by introducing the \pm symbol. In these cases, the optimal control problem can be written as:

$$
\min _{\underline{u}} \int_{t_{0}}^{t_{f}}\left(f(x, u, t) \pm f_{x}^{\prime} y\right) d t
$$

with

$$
\begin{gathered}
\dot{x}=a(x, u, v) \quad \text { and } \quad \dot{y}=A_{x} y+A_{v} w \\
x\left(t_{0}\right)=x_{0}, \quad x\left(t_{f}\right)=x_{f}, \quad y\left(t_{0}\right)=y_{0}
\end{gathered}
$$

Then introducing the two different Hamiltonian functions given by:

$$
H=f(x, u, t) \pm f_{x}^{\prime} y+\lambda^{\prime} a(x, u, v, t)+\mu^{\prime}\left(A_{x} y+A_{v} w\right)
$$

where $\lambda$ and $\mu$ are dual variables with values in $R^{n}$, we get the necessary conditions for an extremal optimal control solution:

$$
\dot{x}=H_{\lambda}(x, u, \lambda, \mu, t), \dot{y}=H_{\mu}(x, u, \lambda, \mu, t), \quad \dot{\lambda}=-H_{x}(x, u, \lambda, \mu, t), \quad \dot{\mu}=-H_{y}(x, u, \lambda, \mu, t)
$$

with the transversality condition $\mu\left(t_{f}\right)=\underline{0}$. 
Let $\left(x^{\min }, u^{\min }\right)$ and $\left(x^{\max }, u^{\max }\right)$ be the solutions of the above optimal control problems to which are attached performance levels written $J^{\min }$ and $J^{\max }$, a fuzzy dual solution of the optimal control problem will be given by:

$$
\tilde{u}(t)=\left(u^{\min }(t)+u^{\max }(t) / 2\right)+\varepsilon\left|u^{\max }(t)-u^{\min }(t)\right| / 2 \quad t \in\left[t_{0}, t_{f}\right]
$$

with an expected fuzzy dual performance given by:

$$
\tilde{J}=\left(J^{\min }+J^{\max } / 2\right)+\varepsilon\left|J^{\max }-J^{\min }\right| / 2
$$

Finally, $\tilde{J}$ can be compared with $J^{\text {mean }}$ to assess the influence of uncertainty in the expected performance.

\section{Conclusion}

In this paper a new approach has been developed to cope with uncertain optimal control problems. First a connection has been established between dual numbers encountered in the design and analysis of kinematics for mechanical systems and triangular fuzzy numbers for which different total orders have been considered. Fuzzy dual functions, fuzzy dual functionals and fuzzy dual differential equations have been introduced, leading to the formulation of fuzzy dual optimal control problems. Adopting a variational approach, fuzzy dual Euler's necessary conditions have been established, then the introduction of a fuzzy dual Hamiltonian allows to establish necessary conditions to be satisfied by the solution of a fuzzy dual optimal control problem. Finally, the fuzzy dual performance of the solution is characterized. The developed approach by handling fuzzy dual numbers presenting only two parameters limits the computational burden associated with fuzzy optimal control problems and should allow to treat large uncertain optimal control problems.

\section{References}

[1] Leitman, G. (1981). The Calculus of Variations and Optimal Control. New York: Springer.

[2] Stengel, R. (1993). Optimal Control and Estimation. Dover publications, New York.

[3] Ying, H. (2000). Fuzzy Control and Modelling: Analytical Foundations and Applications. Wiley-IEEE Press,

[4] Farhadinia, B. (2014). Pontryagin's minimum principle for fuzzy optimal control problems. Iranian Journal of Fuzzy Sets, 11(2), 27-43.

[5] Fisher, I. S. (1999). Dual-Number Methods in Kinematics, Statics and Dynamics. CRC Press.

[6] Cosenza, C. A. N., et al. (2016). Introduction to Fuzzy Dual Mathematical Programming, Fuzzy Systems and Data Mining II, Frontiers in Artificial Intelligence and Applications. IOS Press.

[7] Cosenza, C. A. N., \& Mora-Camino, F. (2016). Fuzzy Dual Numbers: Theory and Applications. COPPE/UFRJ.

[8] Messelmi, F. (2013). Analysis of dual functions. Annual Review of Chaos Theory, Bifurcations and Dynamical Systems, 4, 37-54,

[9] Kirk, D. E. (2004). Optimal Control Theory: An Introduction. Mineola, New York: Dover Publications.

[10] Mora-Camino, F., \& Faye, R. M. (2017). Commande Optimale: Approche Variationnelle. 


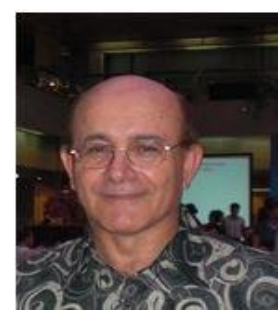

Felix Mora-Camino got the MSc in aeronautics, Dr. Ing. in control and signal processing, the D.Sc. in operations research from Toulouse University, before coming to the French Civil Aviation Institute-ENAC at Toulouse, France, was with COPPE/UFRJ, Rio de Janeiro, Brazil. Presently he is the head of the Automation Research Group at ENAC Research Laboratory and remains an associate researcher at Labfuzzy/COPPE/UFRJ. $\mathrm{Mr}$ Mora-Camino received the grade of Chevalier des Palmes Académiques from the French Higher Education Ministry in 2012.

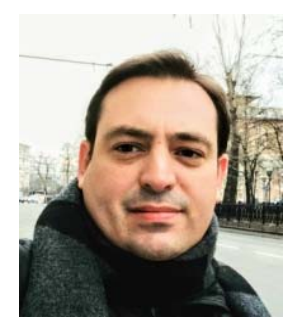

air transport.

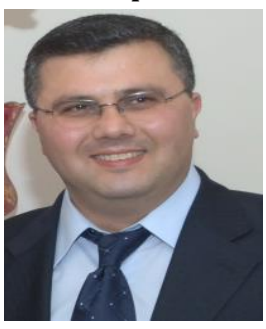

Fabio Krykhtine is a researcher at LABFUZZY COPPE UFRJ where he develops algorithms based on fuzzy logic for decision-making and optimization for widely applications. He became recognized by developing a tool to understand customer's desire applied to fashion industry with emphasis in predict wholesale and retail performance. He is an associated visitor researcher at MAIAA Laboratory, at ENAC France, where he develops research for fuel savings and optimal aircraft trajectories in

Walid Moudani is with the Department of Business Information Systems (BIS) at Lebanese University. He got the MSc and PhD in computer science from Institut National Polytechnique of Toulouse (INPT) and Laboratory for Analysis and Architecture of Systems LAAS-CNRS in 1997 and 2001, respectively. He published more than 50 scientific papers in the areas of optimization, image recognition, compression and analysis, data mining and its applications. He is one member at Group of Bioinformatics and Modeling in the research center of AZM for research in biotechnology and its applications in the Lebanese University (LU).

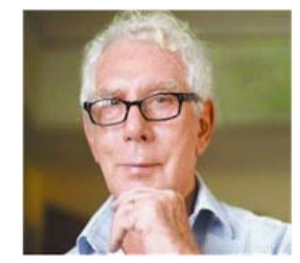

Carlos Alberto Nunes Cosenza is an emeritus professor at COPPE UFRJ and head at LABFUZZY COPPE UFRJ. He developed a hierarquical model to organize industrial location and economic zones called COPPE COSENZA which was applied to the Brazilian Biodiesel Program. Cosenza's work is a reference for many researchers that applied his model in a large variety of knowledge areas, including: production, air transport, ergonomics, medicine, psychology, marketing, economy and industrial location. Cosenza is a visitor researcher at Cambridge where develops propositions for evaluating environmental aspects in new industrial locations scenarios. 